\title{
A model for the indentation size effect in polycrystalline alloys coupling intrinsic
}

\section{and extrinsic length scales}

\author{
Simon P.A. Gill and Christopher J. Campbell \\ Department of Engineering, University of Leicester, \\ University Road, Leicester, LE1 7RH, UK
}

Corresponding author: spg3@leicester.ac.uk (S.P.A. Gill)

\section{Abstract}

The measured hardness of a metal crystal depends on a variety of length scales. Microstructural features, such as grain size and precipitate spacing, determine the intrinsic material length scale. Extrinsic (test) length scales, such as the indentation depth, lead to the indentation size effect (ISE), whereby it is typically found that smaller is stronger. Nix and Gao

${ }^{1}$ developed a widely used model for interpreting the ISE based on forest hardening in single crystalline pure metals. This work extends that model to consider the hardness of polycrystals and alloys, as well as introducing a finite limit to the hardness at very small extrinsic length scales. The resulting expressions are validated against data from the literature. It is shown that a reasonable estimate of the intrinsic material length scale can be extracted from a suite of hardness tests conducted across a range of indentation depths using spherical indenters of various radii. 


\section{Introduction}

The measured hardness of a metal crystal depends on both the intrinsic and extrinsic length scales concerned ${ }^{2-4}$. The intrinsic length scale of a material is governed by the size of the features within its microstructure. Extrinsic length scales arise from the specific nature of the conditions used for the hardness test. In cantilever beam tests and pillar compression tests the extrinsic length scale is typically the dimensions of the specimen itself. In hardness measurements the extrinsic length scale is typically the indentation depth or the radius of the indenter. For flat punch indentations it is a mixture of both [C.J. Campbell and S.P.A. Gill, 2018, unpublished]. The indentation size effect (ISE) is an experimentally observed phenomenon in which the measured hardness of a material is found to increase as the extrinsic length scale decreases. Nix and Gao ${ }^{1}$ first proposed a model to explain this behaviour based on forest hardening due to the presence of geometrically necessary dislocations (GNDs). These are dislocations that are required to accommodate the plastic strain gradients within a crystal. The shear yield stress is assumed to be

(Error!

$$
\tau_{Y}=\beta_{D} G b \sqrt{\rho}
$$

Bookmark not

defined.1)

where $G$ is the shear modulus of the material, $b$ is the magnitude of the Burgers vector, and $\beta_{D}$ is a dimensionless parameter which represents the strengthening contribution due to dislocation hardening. The total dislocation density, $\rho=\rho_{s}+\rho_{G}$, is assumed to be the sum of a constant statistically-stored dislocation (SSD) population of density $\rho_{S}$, and a locally 
evolving population of GNDs, $\rho_{G}$, derived from the plastic strain gradient. The size-dependent hardness of the material is then expressed as

(Error!

$$
H=3 \sqrt{3} \tau_{Y}=H_{0} \sqrt{1+\frac{\rho_{G}}{\rho_{S}}}
$$

\section{Bookmark}

not

defined.2)

where $H_{0}=3 \sqrt{3} \beta_{D} G b \sqrt{\rho_{S}}$ is the macroscopic hardness. GNDs are required to form the numerous surface steps of height $b$ that accommodate the shape of the plastic indent. The number of surface steps (and hence GNDs) are readily determined from the slope of the indenter profile if the contribution from elastic deformation is ignored.

Nix and Gao ${ }^{1}$ derived a model for the ISE for a self-similar (pointed) indenter. Assuming a conical shape with internal angle $\theta$ to the horizontal, the GND density is found to be $\rho_{G}=$ $\frac{3 \tan ^{2} \theta}{2 b h}$. Equation (2) then gives the widely adopted expression

$$
H=H_{0} \sqrt{1+\frac{h^{*}}{h}}
$$

where $h$ is the indentation depth and $h^{*}=\frac{3 \tan ^{2} \theta}{2 b \rho_{S}}$ depends on the SSD density ${ }^{1}$. This analysis was later extended to spherical indenters by Swadener, George and Pharr ${ }^{5}$ for which an 
entirely different scaling behaviour is predicted. For a spherical indenter of radius $R$, the GND density $\rho_{G}=\frac{1}{b R}$ is independent of the indentation depth, such that equation (2) becomes

$$
H=H_{0} \sqrt{1+\frac{R^{*}}{R}}
$$

where $R^{*}=\frac{1}{b \rho_{S}}$.

Equations (3) and (4) have been successfully adopted to model the ISE in many material systems ${ }^{6}$. However, there are a few major shortcomings of this approach. The intrinsic material length scale due to dislocation spacing is included, but the effects of other material length scales, such as grain size, precipitate size and solid solution element spacing are not. Equation (4) assumes the existence of a constant dense population of SSDs with which the GNDs interact, but which is commonly not observed. The hardness is also unphysically predicted to be infinite as the extrinsic length scale tends to zero. These issues are addressed in the following analysis. Section 2 introduces the concept of intrinsic length-scale plasticity beyond just forest hardening. Intrinsic and extrinsic length scale enabled hardness models are then derived based on this concept in section 3. These are shown to reduce to equations (3) and (4) in the limit where the only strengthening mechanism is forest hardening as expected. The models are used to analyse hardness data from the literature in section 4 in a variety of different cases, with the final case demonstrating how the effective intrinsic length scale of a material can be extracted from a series of spherical indentation tests across different indentation depths and indenter sizes. 


\section{Intrinsic material length scales and plasticity}

Dislocation lines moving within a crystal structure are hindered by microstructural obstacles. These obstacles can be roughly classified by their dimensionality: solute atoms (OD), dislocations (1D), grain boundaries (2D) and precipitates (3D) ${ }^{7-10}$. For a dislocation to overcome an obstacle and 'escape' it requires a critical shear stress, $\tau_{C}$, known as the Orowan stress ${ }^{10}$ equivalent to

$$
\tau_{c}=\beta \frac{G b}{L}
$$

where $L$ is the distance between the obstacles and $\beta$ is a mechanism-dependent strengthening factor ${ }^{7,10-14}$. The intrinsic material length scales of a crystal are divided into two categories. Three of the obstacle types, dislocations, solute atoms and precipitates, are classed as by-passable. This is because dislocations can get past them if the stress is high enough. As grain boundaries completely contain a dislocation, they are considered to be not by-passable. Note that the Hall-Petch relationship can be classically derived in two different ways, one through the assumption of forest hardening of grains, which assumes dislocations remain within a grain and multiply to accommodate the applied shear strain, and one which looks at the critical stress required to be produced by a pile-up of dislocations at a grain boundary to promote slip in an adjacent grain ${ }^{9}$. This paper adopts the former approach, consistent with the assumption of boundaries as non-bypassable objects, although this assumption it is not considered critical to this derivation, as both approaches give the same inverse root grain size dependency. Forest (or Taylor) hardening depends on the average spacing between dislocations, $L_{D}=1 / \sqrt{\rho}$, where $\rho$ is the dislocation density. This is assumed to be the only material length scale that changes during plastic deformation and leads directly to equation (1). Solid solution strengthening depends on the average spacing between solute 
atoms. This is taken to be $L_{S}=1 / c^{n}$ where $c$ is the solute strengthening element concentration and $n$ can be either $\frac{1}{2}$ for dilute concentrations or $\frac{2}{3}$ for higher concentrations 9 , 15, 16. Precipitation hardening depends upon the mean spacing between precipitates. If these are assumed to be spherical then it is reasonable to assume that $L_{P}=1.6 r_{P}\left(\frac{\sqrt{\pi}}{4 f}-1\right)$, where $r_{p}$ is the mean radius of the precipitate and $f$ is the volume fraction of precipitates ${ }^{17}$. The contributions from these three stress hardening mechanisms are combined by taking the average effective spacing between the whole field of obstacles, $L_{\tau}$, such that the total shear yield stress is

$$
\tau_{Y}=\tau_{0}+\frac{G b}{L_{\tau}}
$$

where the additional intrinsic (friction) strength of the crystal, $\tau_{0}$, has been introduced. If the width of the obstacles is ignored, the average spacing between them is inversely proportional to their number density. The total number density of obstacles is therefore proportional to $L_{\tau}^{-1}=L_{D}^{-1}+L_{S}^{-1}+L_{P}^{-1}{ }^{11}$. Each type of obstacle has a different level of effectiveness in blocking dislocation movement. Therefore, in practice, each mechanism is weighted accordingly such that the effective length scale is actually taken to be

$$
\frac{1}{L_{\tau}}=\frac{\beta_{D}}{L_{D}}+\frac{\beta_{S}}{L_{S}}+\frac{\beta_{P}}{L_{P}}
$$

where $\beta_{S}=0.01-0.05, \beta_{D}=0.1-0.3$ and $\beta_{P}=0.5-0.8$ are the estimated range of values for the strengthening factors for solid solution strengthening, forest hardening and precipitate hardening respectively ${ }^{9}$. The actual value of $\beta_{i}$ depends on the properties of the 
material, the distribution of obstacles, and the size of the obstacle, e.g. $\beta_{P}$ will be reduced if the precipitate size is too small to prevent precipitates being "by-passed" by cutting. Other methods than (7) have been proposed for combining strengthening contributions, principally root mean squared approaches when the length scales are similar ${ }^{18}$.

The contribution from grain size strengthening is still to be introduced. The grain diameter, $d$, does not appear directly in (7) because grain boundaries are not by-passable obstacles in the Orowan sense. This hardening contribution to (6) must therefore be associated with the dislocation spacing as this is the only length scale that can change during deformation. This will be considered in the next section.

\section{A hardness model with intrinsic and extrinsic length scales}

The hardness model is initially developed for an indenter of general shape and then later explicitly derived for the two cases of self-similar and spherical indenters. The surface profile of the plastic indent is assumed to be commensurate with that of the indenter itself. The indent profile is assumed to be axisymmetric and defined by the indentation depth function, $\delta(r)$, a function of the horizontal radial distance $r$, as shown in Figure 1. For the conceptual purposes of this derivation, it is assumed that GNDs are injected downwards from the surface beneath the indenter during deformation ${ }^{19}$. GNDs are necessary to create the atomic steps that form the plastic indent profile. In practice GNDs may nucleate below the surface and move upwards to form the steps, but this does greatly affect the conclusions of the model. Following Nix and $\mathrm{Gao}^{1}$, the plastic zone beneath the indenter is assumed to be a hemisphere whose radius is the contact radius of the indenter, $a$, defined by $\delta(a)=0$. A number of authors have shown that the radius of the plastic region is in fact larger than the contact 
radius. Durst, Backes and Göken ${ }^{20}$, for instance, amended Nix-Gao using the assumption that the plastic zone radius is related to the contact radius $a_{p z}=f_{a} a$, where the scaling factor can be in the range of $f_{a}=0.5-3.5$. The profile of the indentation defines the distance, $s$, between surface steps of height $b$ to be

$$
\frac{1}{s(r)}=-\frac{1}{b} \frac{d \delta}{d r}
$$

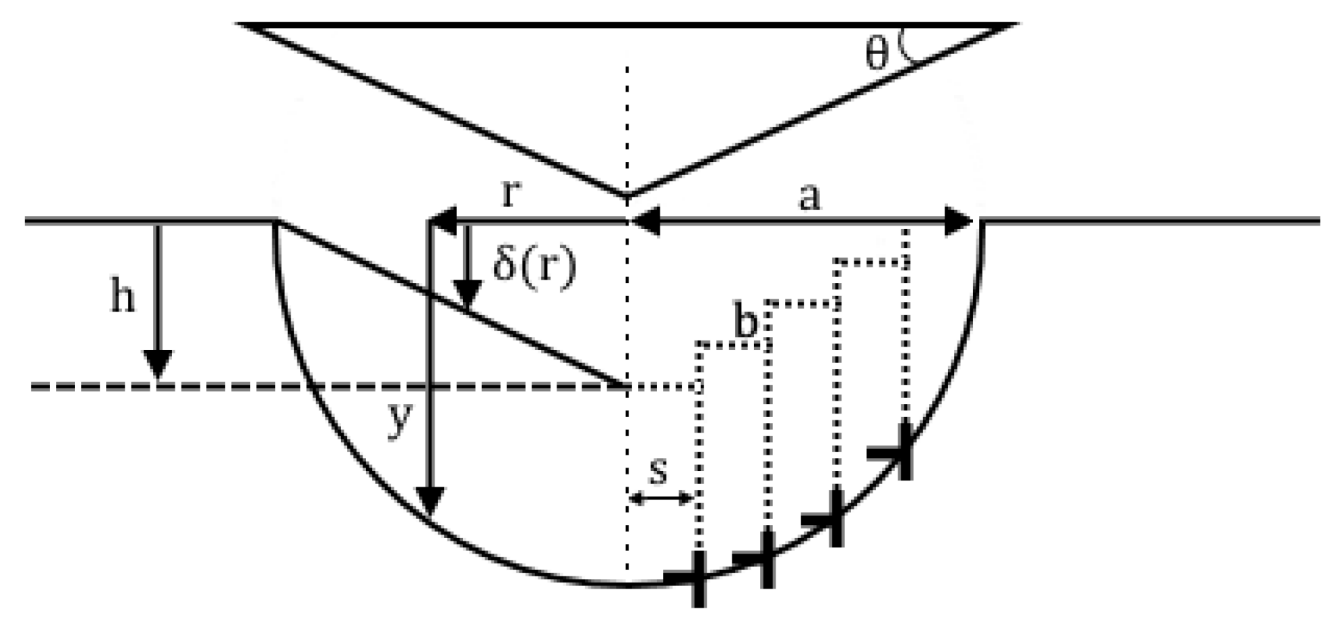

Figure 1: The residual indentation profile in a substrate due a self-similar (conical) indenter after indentation and retraction.

A circular surface step of height $b$ at radius $r$ will generate a GND with a line length of $l=2 \pi r$. This is assumed to travel to the perimeter of the plastic zone, to a depth below the original reference surface $y$. For a hemispherical plastic zone of radius $a$ we have $r^{2}+y^{2}=a^{2}$. The dislocation line density due to a single GND at radius $r$ can be described by

$$
\rho_{G}(r)=\frac{l}{V}=\frac{1}{s(r) y(r)}
$$


where each of the dislocation line loops of length $l$ injected from the surface are contained within a thin hollow cylinder of radius $r$, thickness $s$ and depth $y$ such that the volume is $V=2 \pi r s y$. The average dislocation density is taken to be

$$
\bar{\rho}_{G}=\frac{1}{\bar{s} \bar{y}}
$$

where $\bar{y}$ is the average depth of the hemispherical plastic zone,

$$
\bar{y}=\frac{1}{\pi a^{2}} \int_{0}^{a} 2 \pi r y d r=\frac{2 a}{3}
$$

given $r d r=-y d y$, and the average step spacing $\bar{s}$ is determined from the indenter profile.

Utilisation of equation (10) generates the standard Nix-Gao models for single crystalline pure metals as expected. Here we make two adaptations to account for the nano-indentation response of polycrystalline alloys. Firstly it is assumed that GNDs cannot freely travel from the surface to a depth $y$ in the presence of obstacles. As a dislocation of length $l$ moves through an array of obstacles it is assumed that an additional dislocation length $\alpha l$ will be created. If the obstacles are an average (vertical) distance $\bar{L}$ apart then the volume they occupy is $2 \pi r s \bar{L}$. These will be SSDs such that the average SSD density will become

$$
\bar{\rho}_{S}=\frac{\alpha}{\bar{s} \bar{L}}
$$

For a combination of different obstacles we follow the same process as (7) and write the effective intrinsic length scale due to strain hardening as 


$$
\frac{1}{L}=\frac{2 \alpha_{G}}{d}+\frac{\alpha_{D}}{L_{D}}+\frac{\alpha_{S}}{L_{S}}+\frac{\alpha_{P}}{L_{P}}
$$

The first term on the right hand side contributes to grain size strengthening, and assumes that the mean free path of a dislocation within a grain is equivalent to its radius, $\frac{1}{2} d$. Note that, when combined with (15), the strengthening effect of grain boundaries conforms to the HallPetch relationship, $\frac{1}{\sqrt{d}}$. It is assumed that dislocations cannot cross grain-boundaries, and that a new dislocation must be generated to further accommodate the deformation. For this reason, we expect that $\alpha_{g} \approx 1$, although we note that the mean length of a random cut through a spherical grain of diameter $d$ is $\frac{2 d}{3}$ (as in equation 11) suggesting values of $\alpha_{G}$ up to 1.5 are not physically unreasonable. The effectiveness of the other obstacles in contributing to strain hardening is expected to follow the order predicted for the stress hardening factors, $\beta_{i}$, such that $\alpha_{G}>\alpha_{P}>\alpha_{D}>\alpha_{s}$. A first initial estimate of $\alpha_{i} \approx \beta_{i}$ would appear to be reasonable, although small obstacles such as solute atoms can be by-passed without significant dislocation multiplication (through the motion of jogs) suggesting that $\alpha_{s} \ll \beta_{S}$. Also, dislocation multiplication due to movement past precipitates is likely to be related to the particle size. Overall we have

$$
\rho=\bar{\rho}_{G}+\bar{\rho}_{S}=\rho_{s 0}+\frac{1}{\bar{s}}\left(\frac{3}{2 a}+\frac{1}{L}\right)
$$

where $\rho_{s 0}$ is the initial SSD density in the material and $a$ and $L$ are the extrinsic and intrinsic length scales. The indenter shape defines $\bar{s}$ and will be determined later. 
From (2) and (14) we can therefore write

$$
H=H_{S P 0}+H_{D} \sqrt{1+\frac{1}{\rho_{S 0} \bar{s}}\left(\frac{3}{2 a}+\frac{1}{L}\right)}
$$

where $H_{D}=3 \sqrt{3} G b \beta_{D} \sqrt{\rho_{S 0}}$ is the hardness contribution from dislocation (forest) hardening and $H_{S P 0}=3 \sqrt{3} G b\left(\frac{\beta_{S}}{L_{S}}+\frac{\beta_{P}}{L_{p}}\right)$ is the contribution from the intrinsic strength of the crystal and solute and precipitate hardening.

Before proceeding further, it is worth noting the Nix-Gao model (3) and (4), as well as (15), predict that the dislocation density tends towards infinity as the contact area tends to zero. This is not physically reasonable. Here we avoid this problem by restricting the maximum dislocation density that can occur in a crystal to a fixed value, $\rho_{\max }$. An upper bound of the estimates for this value must be $\rho_{\max } \ll \frac{1}{\boldsymbol{b}^{2}}=16 \times 10^{18} \mathrm{~m}^{-2}$ for $b=0.25 \mathrm{~nm}$, as this represents the (impossible) scenario of a dislocation at every atomic site. Hence it is typically assumed here that $\rho_{\max }=10^{15}-10^{16} m^{-2}$ is the expected range. If we have two lengths, $x$ and $y$, then we can approximate the maximum of these two values using $\max (x, y) \approx$ $\left(x^{n}+y^{n}\right)^{\frac{1}{n}}$ for $n>1$, where the approximation becomes exact as $n \rightarrow \infty$. Here we replace the dislocation spacing $L_{D}=1 / \sqrt{\rho}$ by

$$
L_{D}=\max \left(\frac{1}{\sqrt{\rho}}, \frac{1}{\sqrt{\rho_{\max }}}\right) \approx \sqrt{\frac{1}{\rho}+\frac{1}{\rho_{\max }}}
$$


where we have taken $n=2$ as a weaker approximation of the maximum value to allow for statistical variations. Huang, Zhang, Hwang, Nix, Pharr and Feng ${ }^{21}$ proposed a two-stage model which effectively took a sharp maximum, i.e. $n=\infty$. The forest hardening term, $\rho / \rho_{S 0}$, is therefore replaced in (2) by $\frac{\rho \rho_{\max }}{\rho_{s 0}\left(\rho+\rho_{\max }\right)}=\frac{\rho}{\rho_{s 0}} \cdot \frac{1}{\left(1+\frac{\rho}{\rho_{\max }}\right)}$. With (14), and assuming that $\rho_{s 0} \ll$ $\rho_{\max }$ we can therefore modify (15) to account for very small indentation effects

$$
H=H_{S P 0}+H_{D} \sqrt{1+\frac{\frac{1}{\rho_{S 0} \bar{s}}\left(\frac{3}{2 a}+\frac{1}{L}\right)}{1+\frac{1}{\rho_{\max } \bar{s}}\left(\frac{3}{2 a}+\frac{1}{L}\right)}}
$$

Equation (17) is now evaluated for two different types of indenter profile, self-similar and spherical.

(i) Self-similar indenters

In this case the indenter is assumed to be conical in shape, although this method is relevant to the analysis of any pointed indenter with a constant slope. The geometry is shown in Figure 2 and is defined by the function $\delta(r)=h-r \tan \theta$, where $h$ is the maximum indentation depth, $\theta$ is the indenter angle and $r$ is the radial distance from the axis of symmetry of the indenter. 


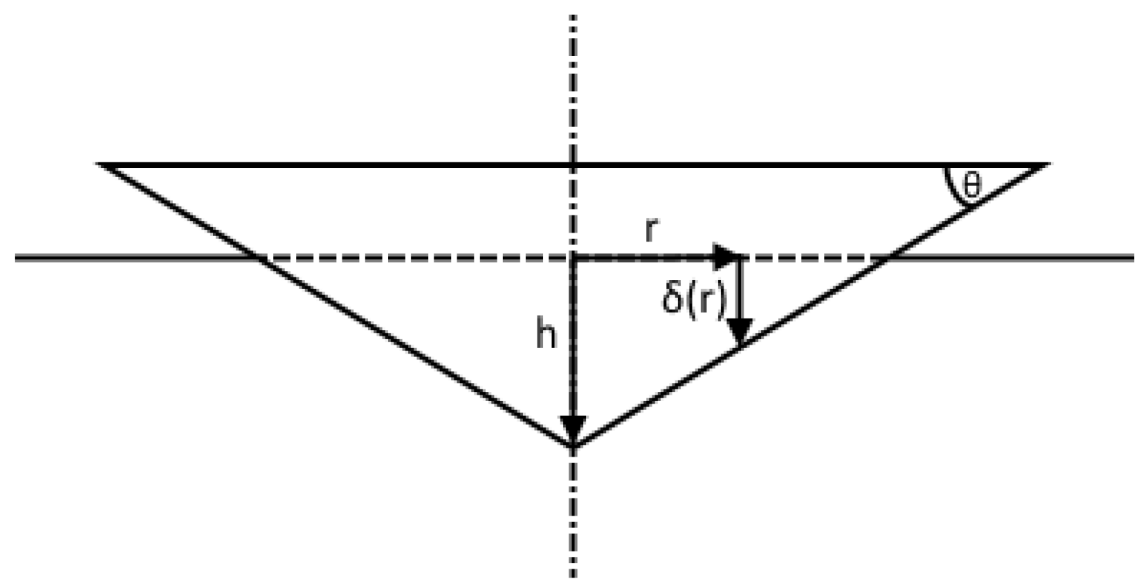

Figure 2: Idealized profile of a self-similar (conical) indent after indentation to a depth $h$ into the surface.

Equation (8) yields

$$
\frac{1}{s}=\frac{\tan \theta}{b}=\frac{1}{\bar{s}}
$$

Noting that $\frac{h}{a}=\tan \theta$ means that substituting (18) into (17) gives the full expression for the ISE for a self-similar indenter as

$$
H=H_{S P 0}+H_{D} \sqrt{1+\frac{\frac{h^{*}}{h}+\frac{L^{*}}{L}}{1+\frac{h_{0}}{h}+\frac{L_{0}}{L}}}
$$

where $h^{*}=\frac{3 \tan ^{2} \theta}{2 b \rho_{s 0}}$ is the Nix-Gao parameter as usual, $h_{0}=\frac{\rho_{s 0}}{\rho_{\max }} h^{*}$ is another fitting parameter and the two other lengths are simply related to these two parameters by $L^{*}=$ $\frac{2}{3 \tan \theta} h^{*}$ and $L_{0}=\frac{2}{3 \tan \theta} h_{0}$. 
It is worth noting at this stage that (19) can be re-written as

$$
H=H_{S P 0}+\widehat{H}_{D} \sqrt{1+\frac{\hat{h}^{*}}{h+\hat{h}_{0}}}
$$

where $\widehat{H}_{D}=H_{D} \sqrt{\frac{f}{e}}, \hat{h}^{*}=e h^{*}+(e-f) h_{0}$, and $\hat{h}_{0}=f h_{0}$ with $e=\frac{L}{L+L^{*}}$ and $f=\frac{L}{L+L_{0}}$. If the intrinsic material length scale $L$ and the indenter angle $\theta$ are constant across all tests to be analysed, then (19) can be written in the same form as (20) with the length scale removed (i.e. $L \rightarrow \infty$ ). This indicates that self-similar indenters cannot be used to uniquely differentiate the intrinsic length scale $L$ from the hardness of a material under these test conditions.

\section{(ii) Spherical indenters}

Figure 3 shows the geometry for a spherical indenter of radius $R$. The indenter contact radius is defined by $a=\sqrt{2 R h-h^{2}} \approx \sqrt{2 R h}$ assuming that the indentation depth $h \ll R$.

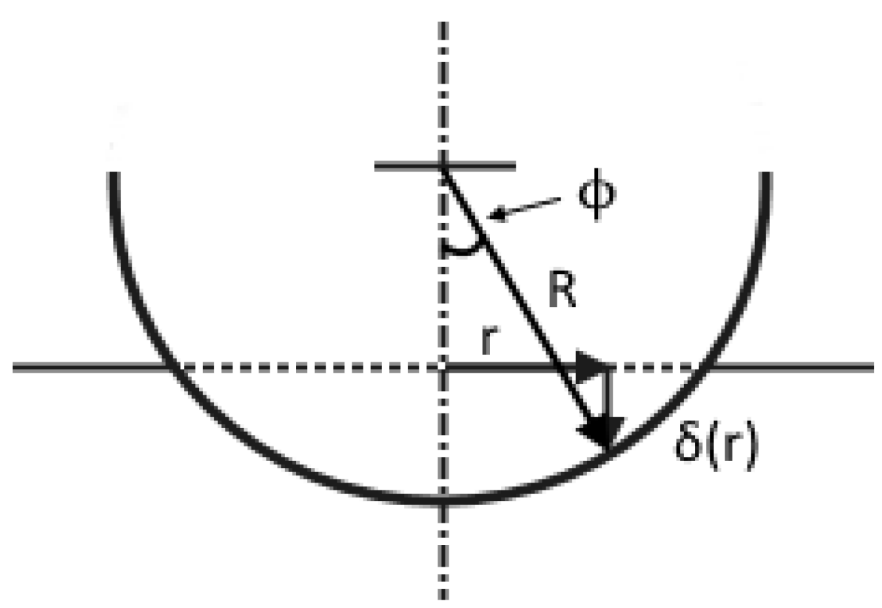

Figure 3: Geometry for a spherical indent.

Equation (8) yields 


$$
\frac{1}{S}=\frac{r}{b \sqrt{R^{2}-r^{2}}} \approx \frac{r}{b R}
$$

giving an average surface step spacing of

$$
\frac{1}{\bar{s}}=\frac{1}{\pi a^{2}} \int_{0}^{a} 2 \pi r \cdot \frac{r}{b R}=\frac{2 a}{3 b R}
$$

Using (22) in equation (17) then provides the full expression for the hardness measured by spherical indenters

$$
H=H_{S P 0}+H_{D} \sqrt{1+\frac{\frac{R^{*}}{R}\left(1+\frac{2 a}{3 L}\right)}{1+\frac{R_{0}}{R}\left(1+\frac{2 a}{3 L}\right)}}
$$

where $R^{*}=\frac{1}{b \rho_{s 0}}$ is the usual Nix-Gao parameter and $R_{0}=\frac{1}{b \rho_{\max }}$.

\section{Validation against published results}

In this section the predictions of the proposed models are tested against data from the literature. The full expressions for the hardness of polycrystalline alloy samples are given in equations (20) and (23) for self-similar and spherical indenters respectively. These equations are simple enough to give practical and physical interpretation of many features of hardness test results. However, (20) and (23) can also generally be simplified for a given situation where a particular mechanism dominates. A number of such sub-cases are considered in this section. Unlike the basic Nix-Gao equations, the proposed hardness functions do not typically allow 
for a selection of plotting axes that allow for a simple linear fit to the data. Hence non-linear regression analysis is performed using the excel Solver function to find the best fit of the model parameters to the data.

\subsection{Hardness of single crystals with intrinsic strength for a self-similar indenter}

The Nix-Gao model for self-similar indenters (3) has been very successful in interpreting experimental hardness data, particularly for single crystalline metals. This model assumes that there is a population of SSDs of density $\rho_{s 0}$ in the crystal which the GNDs, generated by the plastic indentation process, interact with. However, the pre-existing dislocation density in these (typically annealed) samples is low. Here the consequences of assuming that the SSD dislocation population is small $\left(\rho_{s 0} \approx 0\right)$ are examined in this context. In this case (20) can be simplified to

$$
H=H_{S P 0}+\frac{A}{\sqrt{h}}
$$

where $A=9 \tan \theta \beta_{D} G \sqrt{\frac{b}{2}}$ and $H_{S P 0}=3 \sqrt{3} \tau_{0}$. For a pure metal the intrinsic material length scale is very large $(L \rightarrow \infty)$ and it is assumed that GND saturation is not reached $\left(\rho_{\max } \rightarrow \infty\right)$. Figure 4 compares the best fit of the standard Nix-Gao model (3) and equation (24) with single crystal copper data, where the grain size was large compared to the indent. It is interesting to note that (24) provides a slightly better fit to the data than (3) and that the principal difference between the two models is in the macro-indentation regime (near the origin in Figure 4 (b)) where (24) extrapolates to a lower macroscale hardness than (3). 
Best fits are obtained for (3) with $h^{*}=1.78 \mu \mathrm{m}$ and $H_{0}=0.56 \mathrm{GPa}$. Given the definitions of these parameters in (3), and taking $G=48 G P a, b=0.25 \mathrm{~nm}$, and $\theta=25^{\circ}$ then yields $\rho_{s 0}=7 \times 10^{14} \mathrm{~m}^{-2}$ and $\beta_{D}=0.33$. This is a high dislocation density, comparable to that found in a cold-worked sample ${ }^{22}$. Typically the dislocation densities found using (3) are high and often close to the maximum value expected $\rho_{\max }$. This is investigated further in section 4.2 .

Best fits for (24) give $H_{S P 0}=0.365 \mathrm{GPa}$ and $A=18.5 \mathrm{GPa} \sqrt{\mathrm{nm}}$ which predict that $\tau_{0}=70 \mathrm{MPa}$ and $\beta_{D}=0.26$ for the value for the intrinsic strength. The intrinsic strength is typically of the order of a 1-3 MPa so this fitting value is unphysically large. In practice it is expected that both the intrinsic strength and the SSD population contribute to the hardening of materials. The conclusion shows that (3) and (24) are quite similar for typical hardness tests, although the physical constants the fitting parameters relate to are quite different. It is difficult to say if one approach is more valid than another on this basis. For the remainder of this analysis, only one of the (almost indistinguishable) curves will be shown, but the physical parameters relating to each fit will be quoted.

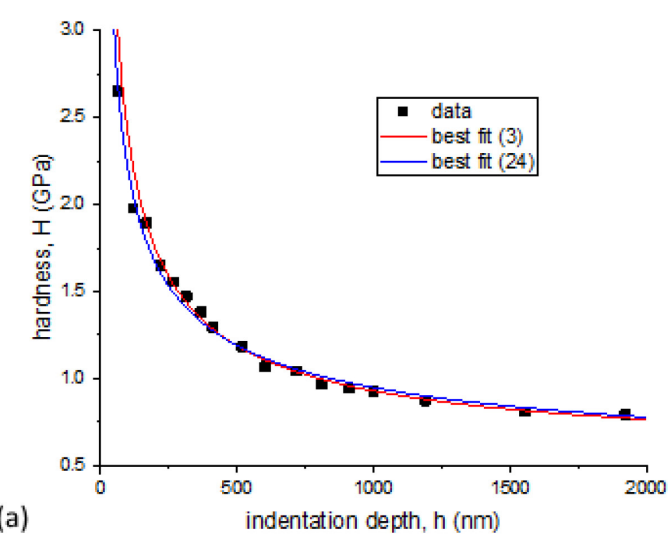

Figure 4: Comparing a forest hardening model (3) against an intrinsic hardening model (24) 
4.2 Hardness of single crystals with a maximum dislocation density for a self-similar indenter

Figure 5 (a) shows $H$ versus $h$ data for electro-polished polycrystalline Iridium with a grain size of $30 \mu m$ using a Berkovich indenter ${ }^{6}$. This is compared in Figure 5 (b) with the Nix-Gao equation (3) which predicts that a plot of $H^{2}$ vs $\frac{1}{h}$ should be linear. It can be seen that this model works for larger scale indents (to the left), but fails at smaller test scales (on the right). Equation (20) is simplified to just include the effect of a maximum dislocation density $\rho_{\max }$ so that

$$
H=H_{D} \sqrt{1+\frac{h^{*}}{h+h_{0}}}
$$

where we take $L \rightarrow \infty$ again for a pure metal and follow Nix-Gao in ignoring strengthening effects not due to forest hardening $\left(H_{S P 0}=0\right)$. This is identical in form to (20) indicating that, for self-similar indenters, (25) also applies to polycrystals as well single crystals. The best fit of (25) in Figure 5 shows that it captures the essential features of the material response much better than equation (3).

The Nix-Gao equation (3) gives $H_{0}=2.2 \mathrm{GPa}$ and $h^{*}=3.48 \mu \mathrm{m}$ yielding predictions of $\rho_{s 0}=3.75 \times 10^{14} \mathrm{~m}^{-2}$ and $\beta_{D}=0.42$ assuming $G=208 \mathrm{GPa}$ for Iridium.

Equation (25) is best fitted by $H_{D}=2.47 \mathrm{GPa}, h^{*}=1.97 \mu \mathrm{m}$, and $h_{0}=150 \mathrm{~nm}$. This yields a prediction of $\rho_{s 0}=6.6 \times 10^{14} \mathrm{~m}^{-2}$ which is about $8 \%$ of the maximum value of $\rho_{\max }=8.75 \times 10^{15} \mathrm{~m}^{-2}$. 
To extend the comparison of section 4.1, if we assume that the initial strength is not due to forest hardening $\left(\rho_{s 0}=0\right)$ then (20) gives

$$
H=H_{S P 0}+\frac{A}{\sqrt{h+\tilde{h}_{0}}}
$$

where $\tilde{h}_{0}=\frac{3 \tan ^{2} \theta}{2 b \rho_{\max }}$. Equation (26) is best fitted when $H_{S P 0}=1.83 \mathrm{GPa}, A=2.43 \mathrm{GPa} \sqrt{\mathrm{nm}}$, and $\tilde{h}_{0}=93 \mathrm{~nm}$. It is not shown in Figure 5 as it is very close to the curve of (25). The theory then predicts that $\tau_{0}=352 \mathrm{MPa}, \beta_{D}=0.25$, and $\rho_{\max }=1.40 \times 10^{16} \mathrm{~m}^{-2}$.

An additional set of test data for single crystal MgO obtained using a Berkovich indenter, shown in Figure 6, also supports this conclusion. Nix-Gao (3) gives $H_{0}=9.3 \mathrm{GPa}$ and $h^{*}=$ $90 \mathrm{~nm}$ yielding predictions of $\rho_{s 0}=1.5 \times 10^{16} \mathrm{~m}^{-2}$ and $\beta_{D}=0.38$ assuming $G=155 \mathrm{GPa}$ for MgO. This indicative SSD dislocation density is very close to the maximum possible density expected.

Equation (25) is best fitted by $H_{D}=9.0 \mathrm{GPa}, h^{*}=180 \mathrm{~nm}$, and $h_{0}=160 \mathrm{~nm}$. This yields a prediction of $\rho_{s 0}=0.75 \times 10^{16} \mathrm{~m}^{-2}$ with $\rho_{\max }=0.84 \times 10^{16} \mathrm{~m}^{-2}$, suggesting that the initial SSD density is almost at the maximum dislocation density, a situation which is most unlikely.

Equation (26) is best fitted when $H_{S P 0}=8.2 \mathrm{GPa}, A=1.45 \mathrm{GPa} \sqrt{\mathrm{nm}}$, and $\tilde{h}_{0}=80 \mathrm{~nm}$. The theory predicts that $\tau_{0}=1.58 \mathrm{GPa}$ and $\rho_{\max }=1.6 \times 10^{16} \mathrm{~m}^{-2}$. Whilst the intrinsic strength is very high, the maximum dislocation density is physically reasonable without requiring an excessive residual SSD density. In practice, the macroscopic hardness will arise from a combination of SSDs and other strengthening mechanisms. This would require using all terms 
in equation (20), but as the two effects are hard to differentiate, a best fit between either of the two cases presented here is valid
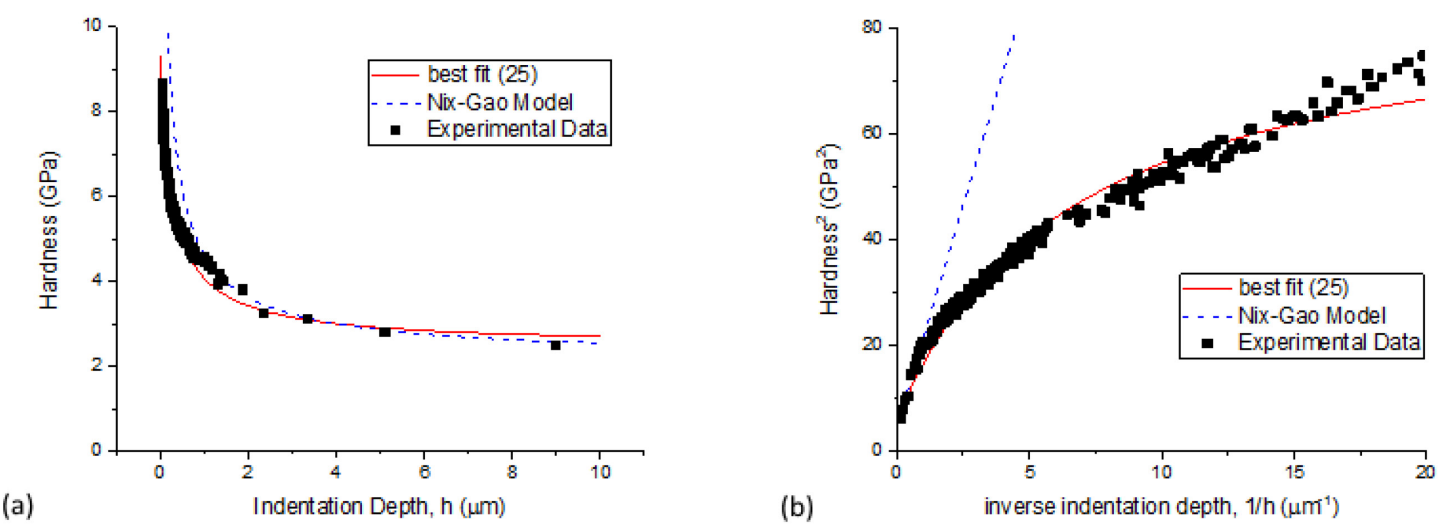

Figure 5: Hardness data as a function of indentation depth for Iridium ${ }^{6}$ for a Berkovich indenter comparing predictions of equations (3) and (25).(a) $H$ vs $h$, and (b) $H^{2}$ vs $\frac{1}{h}$.

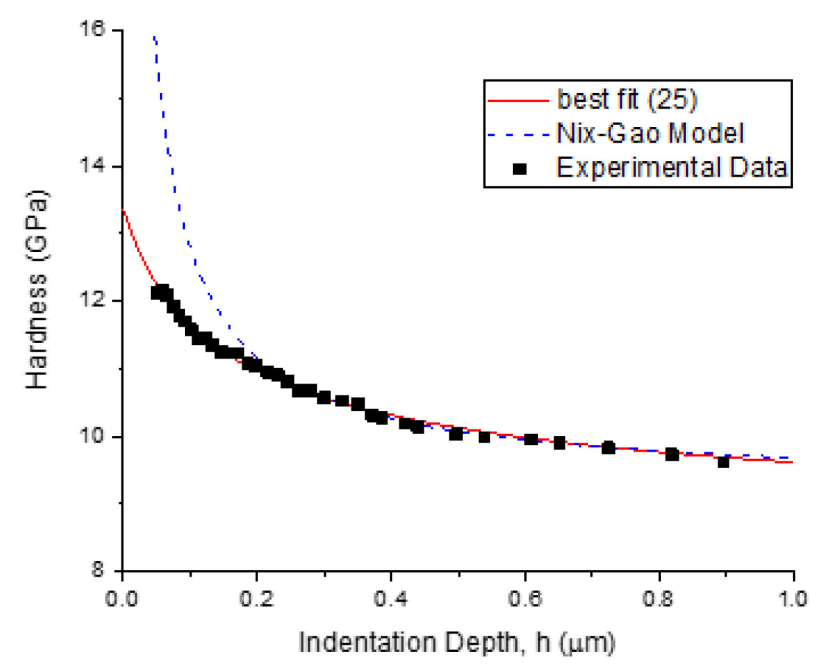

Figure 6: Hardness data as a function of indentation depth for $\mathrm{MgO}^{24}$ for a Berkovich indenter comparing predictions of equations (1) and (25) for $H$ vs h. 
Hou, Bushby and Jennett ${ }^{25}$ have published hardness data for the spherical indentation of copper with different grain sizes. In this case the intrinsic material length scale (grain size) is known but varies from sample to sample. Here we model this data taking the minimum number of degrees-of-freedom required to capture the response, such that intrinsic strengthening $\left(H_{S P 0}=0\right)$ and GND saturation $\left(R_{0}=0\right)$ are ignored. In this case equation the full expression for spherical indentation hardness (23) can be simplified to

$$
H=H_{D} \sqrt{1+\frac{R^{*}}{R}\left(1+\frac{2 a}{3 L}\right)}
$$

Figure 7 shows results of the best fit. The four curves are defined by (27) for a single set of parameters by minimising the mean square error with the data across all four different indenter sizes simultaneously. In this case the hardness is measured at a fixed strain of $\frac{a}{R}=0.25$ and the intrinsic material length scale is taken to be $\frac{1}{L}=\frac{2 \alpha_{G}}{d}$, where $d$ is the measured grain size. An important feature of these hardness measurements is that the indent is positioned within the centre of a grain. If the positioning is random, then the grain size can influence the hardness even if the indentation depth is very much less than $d$. The best fit parameters are $H_{D}=309 M P a, R^{*}=21.3 \mu m$, and $\alpha_{G}=1.41$. It can be seen from Figure 7 that (27) fits the data well. The value of $\alpha_{G} \approx 1$ in line with expectation, given the variation in grain shape and the approximations with the model and the microstructural measurements. The values predict that $\rho_{s 0}=1.88 \times 10^{14} \mathrm{~m}^{-2}$ and $\beta_{D}=0.36$. An equally good fit (not shown) can be achieved assuming intrinsic strengthening such that 


$$
H=H_{S P 0}+\tilde{A} \sqrt{\frac{1}{R}\left(1+\frac{2 a}{3 L}\right)}
$$

where $\tilde{A}=3 \sqrt{3} \beta_{F} G \sqrt{b}$. The best fit is obtained when $H_{S P 0}=0.18 G P a, A=1.21 G P a \sqrt{\mu m}$ and the intrinsic length scale factor $\alpha_{G}=1.45$ similar to before. This predicts that $\tau_{0}=35 M P a$ and $\beta_{D}=0.31$

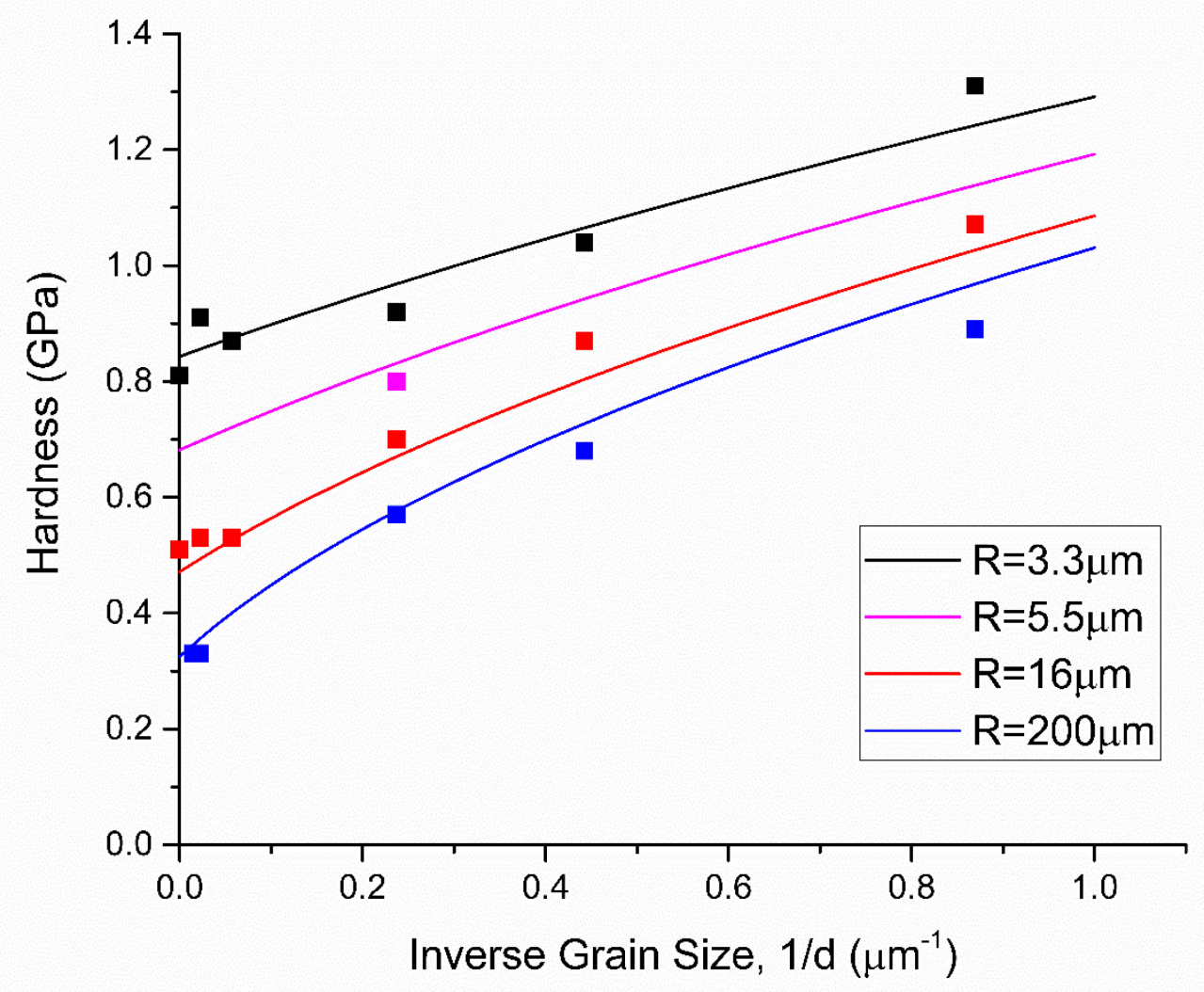

Figure 7: Hardness data for four differently sized spherical indenters as a function of the grain size in polycrystalline copper ${ }^{25}$ compared with a fit from (27). The strain is fixed such

$$
\text { that } \frac{a}{R}=0.25 \text {. }
$$

4.4 Hardness of a polycrystalline alloy with a maximum dislocation density using a spherical indenter 
Swadener, George and Pharr ${ }^{5}$ published data on indentation of a polycrystalline iridium alloy using a range of small indenter sizes. Unlike section 4.3, the intrinsic material length scale in this case is the same for all indents. This is therefore now a fitting parameter to be determined and compared with microstructural observations. For the analysis of this data, we initially ignore strengthening mechanisms in (23) to write

$$
H=H_{0} \sqrt{1+\frac{\frac{R^{*}}{R}\left(1+\frac{2 a}{3 L}\right)}{1+\frac{R_{0}}{R}\left(1+\frac{2 a}{3 L}\right)}}
$$

The best simultaneous fit for the suite of five curves for different indenter radii is shown in Figure 8. This is achieved for $H_{0}=0.21 \mathrm{GPa}, R^{*}=6.0 \mathrm{~mm}, R_{0}=26 \mu \mathrm{m}$, and $L=12.5 \mu \mathrm{m}$. These values predict that $\rho_{S 0}=6.7 \times 10^{11} \mathrm{~m}^{-2}, \rho_{\max }=1.5 \times 10^{14} \mathrm{~m}^{-2}$, and $\beta_{D}=0.95$. The latter is a larger value than expected as is the value of $R^{*}$. This may be due to increased scatter in the hardness measurements for very small spherical indenter radii.

The grain radius was measured by Swadener, George and Pharr ${ }^{5}$ as $\frac{1}{2} d=15 \mu m$. This compares very favourably with the predicted intrinsic material length scale of $L=12.5 \mu \mathrm{m}$. The iridium is also alloyed with $0.3 \% \mathrm{~W}$. This indicates a solid solution strengthening spacing of $L_{S}=5 \mathrm{~nm}$. The two length scales can be combined through (13) such that $\frac{1}{L}=\frac{2 \alpha_{G}}{d}+\frac{\alpha_{S}}{L_{S}}$. It is not possible to separate the contribution from each mechanism but this suggests values of $\alpha_{G}<1.2$ and $\alpha_{s}<4 \times 10^{-4}$, both of which are realistic. 


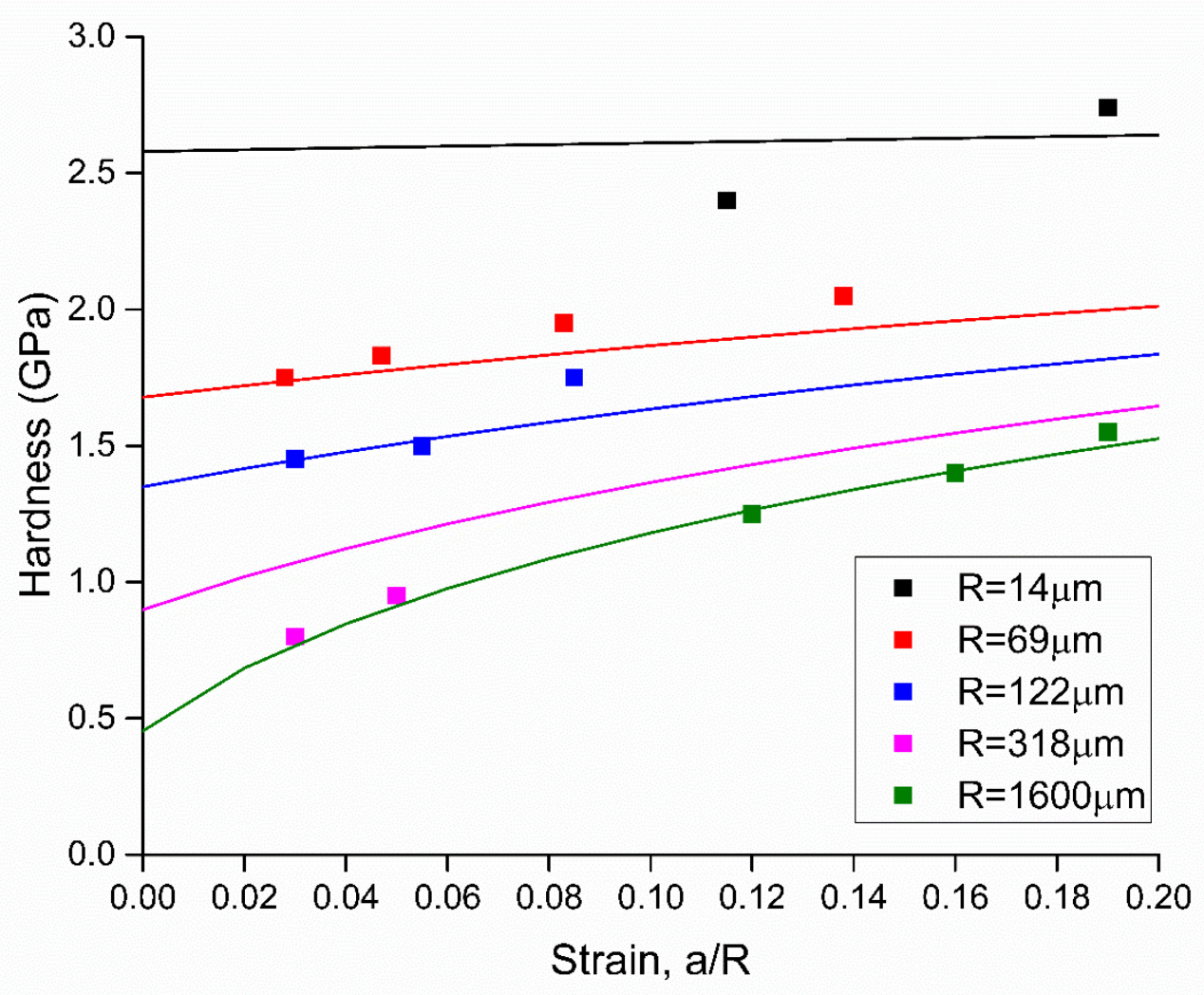

Figure 8: Hardness versus strain for polycrystalline Iridium alloy ${ }^{6}$ for a wide range of spherical indenter radii compared with a fit of equation (29).

\section{Conclusion}

Equations (20) and (23) have been derived for the interpretation of hardness measurements in polycrystalline alloys. These also propose a model for the extreme ISE at very small extrinsic length scales. Validation against data from the literature is very encouraging. It has been demonstrated that a good estimate of the internal material length scale can be extracted from hardness tests using spherical indenters of different radii. It is anticipated that this will be useful to experimentalists in the physical interpretation of data. 


\section{Acknowledgements}

This work was performed as part of a EURAMET joint research project (StrengthABLE) with funding from the European Community's Seventh Framework Programme, ERA-NET Plus, under Grant Agreement No. 217257. 


\section{References}

1. W.D. Nix and H. Gao: Indentation size effects in crystalline materials: A law for strain gradient plasticity J. Mech. Phys. Solids. 46, 411 (1998).

2. J.R. Greer and J.T.M. De Hosson: Plasticity in small-sized metallic systems: Intrinsic versus extrinsic size effect Prog. Mater. Sci. 56, 654 (2011).

3. A.J. Bushby and D.J. Dunstan: Plasticity size effects in nanoindentation J. Mater. Res. 19, 137 (2003).

4. G.M. Pharr and W.C. Oliver: Nanoindentation of silver-relations between hardness and dislocation structure J. Mater. Res. 4, 94 (1988).

5. J.G. Swadener, E.P. George and G.M. Pharr: The correlation of the indentation size effect measured with indenters of various shapes J. Mech. Phys. Solids. 50, 681 (2002).

6. G.M. Pharr, E.G. Herbert and Y. Gao: The Indentation Size Effect: A Critical Examination of Experimental Observations and Mechanistic Interpretations Annu. Rev. Mater. Res. 40, 271 (2010).

7. B. Ehrler, D.J. Dunstan, T.T. Zhu, X.D. Hou, K.M.Y. P'ng and A.J. Bushby: The strength of thin films, small structures and materials under localised stresses Thin Solid Films. 517, 3781 (2009).

8. T. Gladman: Precipitation hardening in metals Mater. Sci. Technol. 15, 30 (1999).

9. J.W. Morris: Dislocation Plasticity: Overview, City, 13/12/2018).

10. T.T. Zhu, A.J. Bushby and D.J. Dunstan: Materials mechanical size effects: a review Mater. Technol. 23, 193 (2008). 
11. B. Ehrler, X.D. Hou, T.T. Zhu, K.M.Y. P'ng, C.J. Walker, A.J. Bushby and D.J. Dunstan: Grain size and sample size interact to determine strength in a soft metal Philos. Mag. 88,3043 (2008).

12. J.R. Greer and W.D. Nix: Nanoscale gold pillars strengthened through dislocation starvation Phys. Rev. B. 73, 245410 (2006).

13. S. Lefebvre, B. Devincre and T. Hoc: Simulation of the Hall-Petch effect in ultra-fine grained copper Mater. Sci. Eng., A. 400-401, 150 (2005).

14. D.J. Dunstan, B. Ehrler, R. Bossis, S. Joly, K.M.Y. P'ng and A.J. Bushby: Elastic Limit and Strain Hardening of Thin Wires in Torsion Physical Review Letters. 103, 155501 (2009).

15. D.R. Jones and M.F. Ashby: Engineering materials 1: An introduction to properties, applications and design (Elsevier, City, 2011).

16. R. Labusch: A Statistical Theory of Solid Solution Hardening physica status solidi (b). 41, 659 (1970).

17. H. Atkinson and S. Gill: Modelling creep in nickel alloys in high temperature power plants, in Structural Alloys for Power Plants, (Elsevier, City, 2014), pp. 447.

18. S. Queyreau, G. Monnet and B. Devincre: Orowan strengthening and forest hardening superposition examined by dislocation dynamics simulations Acta Mater. 58, 5586 (2010).

19. M. Rester, C. Motz and R. Pippan: Where are the geometrically necessary dislocations accommodating small imprints? J. Mater. Res. 24, 647 (2008).

20. K. Durst, B. Backes and M. Göken: Indentation size effect in metallic materials: correcting for the size of the plastic zone Scr. Mater. 52, 1093 (2005).

21. Y. Huang, F. Zhang, K.C. Hwang, W.D. Nix, G.M. Pharr and G. Feng: A model of size effects in nano-indentation J. Mech. Phys. Solids. 54, 1668 (2006). 
22. T.G.d. Sousa, V.L. Sordi and L.P. Brandão: Dislocation Density and Texture in Copper Deformed by Cold Rolling and Ecap Mater. Res. 21, (2018).

23. K.W. McElhaney, J.J. Vlassak and W.D. Nix: Determination of indenter tip geometry and indentation contact area for depth-sensing indentation experiments J. Mater. Res. 13, 1300 (1997).

24. G. Feng and W.D. Nix: Indentation size effect in MgO Scr. Mater. 51, 599 (2004).

25. X.D. Hou, A.J. Bushby and N.M. Jennett: Study of the interaction between the indentation size effect and Hall-Petch effect with spherical indenters on annealed polycrystalline copper J. Phys. D: Appl. Phys. 41, 074006 (2008). 


\section{List of Figures}

Figure 9: The residual indentation profile in a substrate due a self-similar (conical) indenter after indentation and retraction.

Figure 10: Idealized profile of a self-similar (conical) indent after indentation to a depth $h$ into the surface.

Figure 11: Geometry for a spherical indent.

Figure 12: Comparing a forest hardening model (3) against an intrinsic hardening model (24) with hardness ISE data for annealed single crystalline copper ${ }^{23}$ (a) $H$ vs $h$, and (b) $H^{2}$ vs $\frac{1}{h}$.

Figure 13: Hardness data as a function of indentation depth for Iridium ${ }^{6}$ for a Berkovich indenter comparing predictions of equations (3) and (25).(a) $H$ vs h, and (b) $H^{2}$ vs $\frac{1}{h}$.

Figure 14: Hardness data as a function of indentation depth for $\mathrm{MgO}^{24}$ for a Berkovich indenter comparing predictions of equations (1) and (25) for $H$ vs h.

Figure 15: Hardness data for four differently sized spherical indenters as a function of the grain size in polycrystalline copper ${ }^{25}$ compared with a fit from (27). The strain is fixed such that $\frac{a}{R}=0.25$.

Figure 16: Hardness versus strain for polycrystalline Iridium alloy ${ }^{6}$ for a wide range of spherical indenter radii compared with a fit of equation (29). 